\title{
LA DOCENCIA UNIVERSITARIA DE LA EPIGRAFÍA Y NUMISMÁTICA EN LOS NUEVOS TÍTULOS DE GRADO EN HISTORIA ${ }^{1}$
}

\author{
EPIGRAPHY AND NUMISMATICS: \\ GRADUATE TRAINING WITHIN THE FRAMEWORK \\ OF THE NEW EHEA-ADAPTED HISTORY DEGREES
}

\author{
MANUEL RAMÍREZ SÁNCHEZ \\ Universidad de Las Palmas de Gran Canaria
}

Resumen: Desde hace varios cursos académicos se han puesto en marcha en España las nuevas titulaciones universitarias adaptadas al Espacio Europeo de Educación Superior y, en particular, los nuevos grados en Historia. Ello ofrece la posibilidad de que los historiadores podamos discutir cuáles han sido los aspectos positivos y negativos de dicha reforma, con el fin de poder valorar hasta qué punto ésta puede beneficiar o perjudicar la evolución de nuestras disciplinas y la formación de los futuros historiadores, particularmente en lo que se refiere a las materias de Epigrafía y Numismática, de larga tradición en las enseñanza universitaria.

Palabras clave: Epigrafía, Numismática, Grados, Espacio Europeo de Educación Superior.

\begin{abstract}
For some years now, new EHEA degrees have begun to flourish in Spain, History studies not being an exception. Reached this stage, historians can now start to debate over the negative and the positive sides to this Higher Education reform program; in addition to this, historians will have the chance to assess its bearance upon other related disciplines and the way future historians will get trained, specially in such fields of knowledge as Numismatics and Epigraphy, both of them boasting a long tradition in our universities.
\end{abstract}

\footnotetext{
${ }^{1}$ Este trabajo se ha realizado en el marco del proyecto de investigación ProID20100213, financiado por el Gobierno de Canarias, a través de la Agencia Canaria de Investigación, Innovación y Sociedad de la Información. Agradezco a Juan Manuel Abascal Palazón, Francisco Beltrán Lloris, Antonio Castillo Gómez, José Manuel Iglesias Gil y Javier de Santiago Fernández la información sobre los planes de estudios de sus respectivas universidades. Una versión reducida de este trabajo fue presentada oralmente en el XIII Congreso Español de Estudios Clásicos, celebrado en julio de 2011 en la Universidad de Logroño. Ante las limitaciones de espacio impuestas para su publicación en las Actas de dicho Congreso, hemos optado por publicar íntegramente el texto en esta revista.
} 


\section{MANUEL RAMÍREZ SÁNCHEZ \\ LA DOCENCIA UNIVERSITARIA DE LA EPIGRAFÍA Y NUMISMÁTICA...}

Keywords: Epigraphy, Numismatics, Degrees, European Higher Education Area.

\section{INTRODUCCIÓN.}

La reflexión teórica sobre la enseñanza y el aprendizaje de las Ciencias Históricas en la universidad española realizada por especialistas ajenos a la especialidad de las didácticas no es, ciertamente, frecuente. A diferencia de lo que sucede en otros países, sobre todo del área anglosajona, en pocas ocasiones los historiadores nos ocupamos del análisis de la estructura, objetivos y fines de nuestra enseñanza, más allá del trámite burocrático y, en cierta medida, lastrado por los usos impuestos por la costumbre, de la presentación de las memorias docentes de los concursos a plazas de Titular y Cátedra y, a partir de las recientes reformas universitarias, incluso en las de algunos profesores contratados ${ }^{2}$. Más allá de ese ejercicio personal, y en ocasiones incluso íntimo, el debate entre los especialistas acerca de qué enseñamos y para qué, no suele ir más allá de las conversaciones entre colegas, salvo algunos casos, en los que se utilizan las páginas de la prensa, más como un vehículo de desahogo personal que con otros fines más ambiciosos.

Ni siquiera en los últimos años, al abrigo del proceso reformista que todavía estamos viviendo en las universidades españolas, los historiadores hemos aprovechado la oportunidad que ofrece el reto de la convergencia europea para realizar un profundo análisis de la necesidad de mejora de nuestros planes de estudio, con el fin de adaptarlos a las necesidades reales de nuestros estudiantes. Existen, no obstante, algunas publicaciones que debemos mencionar aquí, surgidas por el debate suscitado en torno a la elaboración del llamado Libro Blanco del Grado en Historia $^{3}$, solo se han publicado algunos trabajos de especialistas en Historia Contemporánea ${ }^{4}$ o de Historia Económica ${ }^{5}$, en parte animados por el debate interno en

\footnotetext{
${ }^{2}$ Un género de literatura gris que, como señalaba hace tiempo Ruiz Zapatero, destaca por mostrar "una absoluta idealización de la enseñanza y en muchos casos, incluso el reverso de la realidad docente”. G. RUIZ ZAPATERO, “Enseñando Arqueología... ¿Hay algo que decir?”, Arqueoweb, 0 (1999) [en línea] <http://www.ucm.es/info/arqueoweb/> [Consulta: 01/10/2012].

3 J. A. CATALÁ et alii, Informe para el diseño del título del Grado de Historia, ANECA, Madrid, 2004 [en línea] < http://www.aneca.es/media/150448/libroblanco_jun05_historia.pdf > [Consulta: 01/10/2012].

${ }^{4}$ C. GARCÍA MONERRIS, "El Espacio Europeo de Educación Superior: otra manera de enseñar y de aprender (La experiencia de la Titulación de Historia de la Universidad de València)”, Ayer, 58 (2005), pp. 307-329; C. GARCÍA MONERRIS, F. GÓMEZ OCHOA, "Los estudios universitarios de historia en España ante la Convergencia Europea”, Ayer, 57 (2005), pp. 295-314.
} 
las asociaciones de profesores de sus respectivas áreas de conocimiento ${ }^{6}$. Salvo las excepciones citadas, el profesorado de las Facultades de Geografía e Historia y de Filosofía y Letras, en su mayoría, ha vivido este proceso desde una absoluta indiferencia, cuando no desde una oposición al proceso reformista. En este contexto no es de extrañar que el denominado proceso de Bolonia, que pretendía la elaboración de unos títulos que permitieran la convergencia de los nuevos grados con los de otros países del Espacio Europeo de Educación Superior, haya propiciado tal heterogeneidad entre los planes de estudio que hace materialmente imposible la propia convergencia de los mismos en el sistema universitario español.

Dicho en otras palabras, la adaptación a Bolonia ha propiciado unos títulos con una distribución de asignaturas e itinerarios curriculares tan heterogénea, que no ha hecho sino aumentar las diferencias que ya existían entre las universidades españolas antes de la reforma, incrementando estas diferencias incluso entre las universidades de una misma Comunidad Autónoma. El resultado final, en el aspecto que aquí nos interesa analizar, es que con los nuevos planes de estudio, un futuro historiador puede obtener su título de graduado en Historia sin haber estudiado unas materias tan esenciales en su formación como son la Epigrafía y Numismática, no ya como obligatorias, ni siquiera como optativas. Después de haber dedicado varios trabajos anteriores a destacar la importancia de la enseñanza universitaria de estas materias ${ }^{7}$ hemos considerado oportuno hacer un balance de cuál

${ }^{5}$ F. J. HERNANDO ORTEGA, "La enseñanza de la Historia Económica en Administración y Dirección de Empresas. Una experiencia docente dentro del Espacio Europeo de Educación Superior”, en J. TURMO (ed.), El camino hacia Bolonia: experiencias de innovación docente, Universidad Autónoma de Madrid, Madrid, 2010, pp. 37-60; A. TONIOLO, M. LLONCH, "La adaptación de la asignatura Historia Económica Mundial en el Espacio Europeo de Educación Superior: objetivos y primeros resultados en la Facultad de Ciencias Económicas y Empresariales de la UAB”, Working Papers, 8 (2007), [en línea] <http://www.h-economica.uab.es/wps/2007_08. pdf $>$ [Consulta: 01/10/2012].

${ }^{6}$ Habría que citar también los artículos publicados en el dossier “¿Qué arqueología enseñar en la universidad del siglo XXI?”, publicado en la revista Complutum. Cfr. J. ÁLVAREZSANCHÍS, “¿Qué arqueología para mañana?”, Complutum, 20/2 (2009), pp. 242-245; X.-L. ARMADA, (2009), “Una arqueología diferente”, Complutum, 20/2 (2009), pp. 246-251.; G. RUIZ ZAPATERO, “¿Qué arqueología enseñar en la universidad del siglo XXI?”, Complutum, 20/2 (2009), pp. 225-238.

7 M. RAMÍREZ SÁNCHEZ, "Recursos de Epigrafía y Numismática en Internet: balance actual y perspectivas en España”, Boletín Millares Carlo, 22 (2003), pp. 275-301; “La utilización de las nuevas tecnologías en la docencia universitaria de la Paleografía, Diplomática, Epigrafía y Numismática: tradición e innovación”, en J. J. CASTRO y J. M. RODRÍGUEZ (eds.), Actas de las II Jornadas Canarias sobre las TIC en la docencia universitaria, Universidad de Las Palmas de Gran Canaria, Las Palmas de Gran Canaria, 2005, pp. 279-288; "Las Tecnologías de la Información y la Comunicación aplicadas a la docencia universitaria en Epigrafía y Numismática: presente y futuro", en Actas del XI Congreso de la Sociedad Española de Estudios Clásicos (Santiago de Compostela, 2003), vol. III, SEEC, Madrid, 2006, pp. 831-841; (2010), "Epigrafía e 


\section{MANUEL RAMÍREZ SÁNCHEZ \\ LA DOCENCIA UNIVERSITARIA DE LA EPIGRAFÍA Y NUMISMÁTICA...}

es la situación actual en la que han quedado estas ciencias historiográficas en nuestras universidades.

\section{EL GRADO EN HISTORIA, BALANCE DESPUÉS DE LA RE-} FORMA.

Desde que en agosto de 1983 se aprobó la Ley de Reforma Universitaria, el sistema universitario español ha experimentado profundos cambios que, en unos casos, han sido más traumáticos que otros. Veinte años más tarde de aquella reforma, que fue muy criticada entre los sectores más conservadores de la sociedad española, las universidades han vivido el proceso de convergencia europea, que tenía fijada en el año 2010 la fecha límite para la implantación de los nuevos títulos de grado. Entre ambas fechas, algunas cifras permiten conocer cómo ha evolucionado el sistema universitario español. En efecto, si en 1983 había 34 universidades en España -de las cuales treinta eran públicas y solo cuatro privadas, todas ellas pertenecientes a la Iglesia católica-, en 2011 la cifra total de universidades se eleva a 78, con cincuenta de ellas de carácter público y veintiocho privadas ${ }^{8}$. Este incremento del número de universidades ha venido acompañado, como es lógico, de un crecimiento del número de estudiantes, que ha pasado de los 743.432 alumnos matriculados en el curso 1983-1984 a 1.556.377 estudiantes del curso 2009$2010^{9}$.

Aunque la cifra total de estudiantes universitarios ha crecido poco en los últimos años, es de destacar que la oferta de nuevos grados y, sobre todo de posgrados, se ha incrementado notablemente. Centrándonos únicamente en los estudios universitarios de Historia, antes del inicio del proceso de Bolonia 34 universidades incluían entre sus titulaciones la Licenciatura en Geografía e Historia o en

Internet en España hoy: una introducción necesaria”, en J. M. IGLESIAS GIL (ed.), Actas de los XX Cursos monográficos sobre el Patrimonio Histórico, Universidad de Cantabria, Santander, 2010, pp. 15-41.

${ }^{8}$ El crecimiento de universidades públicas casi ha duplicado la cifra de 1983, al pasar de treinta a cincuenta. Sin embargo, el número actual de universidades privadas multiplica por siete la cifra de 1983. Si analizamos los datos por Comunidades Autónomas, podemos observar que la Comunidad de Madrid es la que más universidades tiene - 15 en total, de las cuales 9 son privadas-. Todas las comunidades, excepto seis (Galicia, Asturias, Cantabria, Castilla La Mancha, Extremadura y Andalucía) compaginan el sistema universitario público con el privado, aunque Andalucía es la que más universidades públicas tiene, en total diez. MINISTERIO DE EDUCACIÓN, Datos y cifras del Sistema Universitario Español, Madrid. Curso 2010-2011, Madrid 2010, p. 4.

${ }^{9}$ MINISTERIO DE EDUCACIÖN, Datos y cifras..., p. 8. 
Historia $^{10}$. Sin embargo, una vez finalizado el proceso de convergencia, esta cifra se ha incrementado notablemente, ya que en la actualidad son 42 las universidades que ofertan un Grado en Historia, con algunas variaciones en su denominación, como veremos más adelante. En efecto, la implantación de los nuevos grados ha sido aprovechada por algunas universidades que no incluían las Ciencias Históricas en su catálogo de titulaciones para ofertar un nuevo grado en Historia. Es el caso, por ejemplo, de la Universidad de Almería, la Universidad de Burgos, la Universidad de Jaén, la Universidad Jaume I, la Universidad de La Rioja, la Universidad Rey Juan Carlos o la Universidad San Pablo-CEU. A estas habría que añadir universidades privadas de reciente creación, que han incluido el Grado en Historia en su oferta de titulaciones, como sucede con la Universidad a Distancia de Madrid. Paradójicamente la Universidad de Deusto, una universidad privada de la Iglesia Católica de gran tradición en el sistema universitario español, ha suprimido la Historia de su catálogo de Titulaciones ${ }^{11}$.

Algunas universidades han aprovechado la reforma para ofrecer un título más especializado, como el Grado en Historia y Patrimonio Histórico que oferta la Universidad de Extremadura, aunque esta apuesta innovadora ha sido impulsada por aquellas universidades que han creado ex novo el Grado en Historia. Es el caso, por ejemplo, de la Universidad de Burgos y la Universidad de Jaume I, que han apostado por crear un nuevo título de Grado en Historia y Patrimonio. Otras universidades, en cambio, han mantenido un título de mayor tradición, como es el Grado en Geografía e Historia. Ésta ha sido la decisión, por ejemplo, de la Universidad Nacional a Distancia y la Universidad de Vigo. Cabe destacar que algunas universidades que, por vez primera, ofertan un título en Historia, también han apostado por un Grado en Geografía e Historia. Es el caso, por ejemplo, de la Universidad de Jaén, la Universidad de La Rioja o la Universidad Pablo de Olavide. Respecto a los grados con un perfil más innovador, de Historia y Patrimonio, a nadie se le escapa que, detrás de su diseño se esconde el interés por aprovechar la sinergia de aquellas ciudades Patrimonio de la Humanidad que son, a su vez, sede de las universidades que ofertan estos títulos ${ }^{12}$.

\footnotetext{
${ }^{10}$ J. A. CATALÁ et al., Informe para el diseño..., p. 31.

${ }^{11}$ El título de Licenciado en Historia, de la Facultad de Filosofía y Letras de la Universidad de la Iglesia de Deusto (BOE de 8 de febrero de 2000), ha quedado reducido a una Mención en Historia en el nuevo Grado en Humanidades, con 48 ECTS más un Trabajo fin de Grado de 6 ECTS (BOE de 19 de diciembre de 2009).

${ }^{12}$ Así, por ejemplo, la Universidad de Burgos o la Universidad de Extremadura. La primera de ellas se enclava en una ciudad cuya catedral gótica y alrededores fue declarada Patrimonio de la Humanidad por la UNESCO en 1984. En el caso de la Universidad de Extremadura, su campus
} 


\section{MANUEL RAMÍREZ SÁNCHEZ \\ LA DOCENCIA UNIVERSITARIA DE LA EPIGRAFÍA Y NUMISMÁTICA...}

Como todos sabemos, el proceso de implantación de los nuevos grados no ha sido fácil y en las universidades hemos vuelto a asistir a interminables discusiones acerca de la estructura de los futuros planes de estudio, así como sobre la inclusión, desaparición o amortización de las asignaturas. A pesar de que la filosofía de esta reforma se basaba no solo en una mera transformación de los planes de estudio, sino en una profunda reestructuración de los métodos de enseñanzaaprendizaje, el resultado que todos conocemos ha tenido más que ver con lo primero que con lo segundo. Así las cosas, conviene analizar cómo han quedado configurados los planes de estudio de los nuevos grados en Historia, con el fin de reconocer cuál es la situación actual, y la proyección futura, de los estudios de Epigrafía y Numismática en el sistema universitario español ${ }^{13}$. La recopilación de la información necesaria para la realización del presente estudio no ha sido fácil ya que muchos planes de estudio aún no se han publicado en el Boletín Oficial del Estado. Por ello hemos procedido a consultar la información disponible en las páginas web de cada una de las universidades ${ }^{14}$.

\section{EL PUNTO DE PARTIDA: LA EPIGRAFÍA Y NUMISMÁTICA EN LAS ANTERIORES LICENCIATURAS EN HISTORIA.}

Con la puesta en marcha de la Ley General de Educación de 1970, durante el mandato del ministro Villar Palasí, se acometió la reforma de las titulaciones universitarias, y entre ellas, las ofertadas por las Facultades de Filosofía y Letras. Nació así, en 1973, la Licenciatura de Geografía e Historia, a partir de las directrices generales elaboradas por la Dirección General de Universidades, que fueron desarrolladas por las correspondientes secciones de Geografía e Historia de las Facultades de Filosofía y Letras de todo el Estado. Con aquel plan de estudios nos formamos en España decenas de miles de Licenciados/as en Geografía e Historia,

histórico y la Facultad de Filosofía y Letras están enclavados en Cáceres, cuyo conjunto urbano fue catalogado como Monumento Nacional en 1949, y en 1986 alcanzó la proclamación de Patrimonio de la Humanidad por la UNESCO. En lo que se refiere a la Universidad Jaume I, la oferta de un Grado en Historia y Patrimonio Histórico debe entenderse más como una apuesta estratégica por ofertar un título bien diferenciado de los que poseen otras universidades de la Comunidad Valenciana y de otras Comunidades Autónomas limítrofes.

${ }^{13}$ En otro trabajo analizaremos cuál es la presencia de la enseñanza de estas materias en la oferta de posgrados oficiales que, presumiblemente, realizarán los graduados en Historia en nuestras universidades.

${ }^{14}$ Todas las consultas en Internet y las referencias a documentación publicada en la web se han revisado con la fecha que se indica en cada caso, entre corchetes, siguiendo la norma para la citación de recursos electrónicos. 
algunos de los cuales tuvimos la fortuna de poder dedicamos a la tarea de enseñar e investigar en la universidad. Aquel plan de estudios constaba de dos ciclos, el primero, de carácter generalista y común, de tres cursos de duración, y el segundo ciclo, de especialización, que constaba de dos cursos. Según la disponibilidad del profesorado existente, unas universidades ofertaron más o menos especialidades dentro de las respectivas secciones (Geografía, Historia, Historia del Arte, etc.), y dentro de cada una de ellas se crearon las materias, de carácter obligatorio -las más- y optativo -las menos-. Entre las materias de carácter obligatorio u optativo, según las distintas especialidades, se encontraban las asignaturas de "Paleografía y Diplomática”, por un lado, y “Epigrafía y Numismática”, por otro.

En los años noventa del pasado siglo, la reforma de las titulaciones universitarias emprendidas a partir de la entrada en vigor de la Ley de Reforma Universitaria, de 1983, significó una profunda alteración de la Titulación de Geografía e Historia que, entre otras cosas, suponía la desaparición de aquella formación común y nacía la especialización, desde el primer curso, de los estudiantes según la opción escogida: Geografía o Historia. Con el desarrollo de los nuevos planes de estudios de la Titulación de Historia aquellas materias hasta entonces independientes como eran "Paleografía y Diplomática” y "Epigrafía y Numismática”, pasaron a convertirse en una única asignatura, de carácter troncal, denominada "Paleografía Diplomática, Epigrafía y Numismática"15. El descriptor que acompañaba su denominación en las directrices generales propias del Título, redactadas por el Consejo de Universidades, destacaba por su excesivo laconismo: "Estudio, interpretación y valoración de las fuentes históricas escritas” y una de las principales consecuencias que se derivaron de la creación de esta materia troncal, fue la unión, dentro de una misma asignatura, de disciplinas tradicionalmente impartidas por profesorado de distintas áreas de conocimiento ${ }^{16}$.

Unos años más tarde, la entrada en vigor del Real Decreto 779/1998 introdujo algunas novedades en la asignatura al aumentar su carga docente, que pasó de los cuatro créditos iniciales a los seis créditos, o lo que es lo mimo, 60 horas lectivas. Esta materia troncal, por lo general situada en el segundo ciclo del plan de estudio, en el momento de redactar estas líneas va camino de su progresiva extinción, en los próximos cursos académicos, en las 34 universidades españolas que han venido ofertando la Licenciatura en Historia.

\footnotetext{
15 Sobre este particular remitimos al exhaustivo estudio de $\mathrm{M}^{\mathrm{a}}$ M. CÁRCEL ORTÍ, La enseñanza de la Paleografía y Diplomática. Centros y cursos, Valencia, 1996, pp. 143 y ss.

${ }^{16} \mathrm{M}^{\mathrm{a}}$ M. CÁRCEL ORTÍ, "La Paleografía y Diplomática en las universidades españolas”, Signo, 9 (2002), pp. 37-104.
} 


\section{MANUEL RAMÍREZ SÁNCHEZ \\ LA DOCENCIA UNIVERSITARIA DE LA EPIGRAFÍA Y NUMISMÁTICA...}

Debido a la escasa carga docente que poseía esta asignatura troncal y, ante la necesidad de que los estudiantes de la Licenciatura de Historia pudieran obtener una formación más amplia en estas materias, la mayoría de las Facultades de Filosofía y Letras y de Geografía e Historia han venido ofertando otras asignaturas, casi en su totalidad optativas, en el segundo ciclo de la licenciatura. Sin ánimo de hacer aquí un listado exhaustivo podemos destacar asignaturas como "Paleografía” (impartida, entre otras, en las universidades de Alcalá, Autónoma de Barcelona, Granada y León), “Paleografía romana” (universidades de Málaga y Oviedo), "Paleografía medieval” (universidades de Alicante, Autónoma de Madrid, Complutense de Madrid y Cádiz, entre otras), “Paleografía de la Edad Moderna” (universidades de Alicante y Autónoma de Madrid), "Paleografía y Diplomática hispánicas de la Edad Media” y "Paleografía y Diplomática hispánicas de la Edad Moderna” (Universidad de Oviedo), "Paleografía y Epigrafía” (Universidad de Valencia), "Paleografía, Diplomática y Numismática” (Universidad de Murcia), "Historia de la cultura escrita" (universidades de Alcalá y Las Palmas de Gran Canaria, entre otras), "Epigrafía y Numismática medievales” (Universidad de Murcia), "Epigrafía y Numismática en el Mundo Clásico” y "Numismática Moderna y Sistema monetario europeo” (Universidad Complutense de Madrid), "Escrituras expuestas: inscripciones y graffiti” (Universidad de Alcalá), entre otras ${ }^{17}$.

\section{LA EPIGRAFÍA Y NUMISMÁTICA EN EL LIBRO BLANCO DEL GRADO EN HISTORIA.}

Nadie discute que la educación universitaria en España ha adolecido de un vicio muy extendido en todas las Facultades y Escuelas: demasiada teoría y poca práctica. En el caso concreto de los estudios de Historia, los planes de estudio han estado sobrecargados de asignaturas cuyo principal objetivo era proporcionar nuevos conocimientos teóricos o profundizar en los ya adquiridos en cursos anteriores. Cuando nadie hablaba aun en nuestras universidades de habilidades y competencias o, a lo sumo, empleaban esa jerga los psicólogos y pedagógos, en nuestras Facultades de Filosofía y Letras y de Geografía e Historia, los estudiantes tenían ocasión de acceder a las fuentes históricas desde los primeros cursos de la Licenciatura. A diferencia de lo que sucedía en otras Facultades, en las que el peso de las clases teóricas era mucho mayor, los estudiantes tenían un buen número

\footnotetext{
${ }^{17}$ Un listado pormenorizado de todas estas asignaturas en $\mathrm{M}^{\mathrm{a}} \mathrm{M}$. CÁRCEL ORTÍ, op.cit., pp. 46-88. No existe un estudio similar para las asignaturas de las materias de Epigrafía y Numismática.
} 


\section{MANUEL RAMÍREZ SÁNCHEZ \\ LA DOCENCIA UNIVERSITARIA DE LA EPIGRAFÍA Y NUMISMÁTICA...}

de clases prácticas en las que el comentario de texto proporcionaba acceso a las fuentes escritas y las proyecciones de diapositivas a las fuentes materiales. Y en el segundo ciclo, según las especialidades que cursaran, los alumnos tenían ocasión de profundizar en su formación como futuros historiadores, aprendiendo los rudimentos de las ciencias específicas que les permitían estudiar las fuentes escritas y materiales.

Respecto a las competencias específicas que debe poseer un historiador, según el perfil profesional, el Libro Blanco destacaba el "conocimiento de y habilidad para usar técnicas necesarias para estudiar documentos de determinados períodos (Paleografía, Epigrafía)”, cuya valoración en una escala de 1 a 4 oscilaba entre el 3,72 para el perfil de investigación y el 2,86 para el de docencia ${ }^{18}$. En el informe se incluyeron las valoraciones de las competencias genéricas y específicas realizadas por diversas asociaciones profesionales, en particular por la Sociedad Española de Estudios Medievales, la Asociación Profesional de Arqueólogos de Galicia, la Fundación Española de Historia Moderna y la Asociación Española de Historia Contemporánea ${ }^{19}$. Tanto los medievalistas como los arqueólogos y modernistas resaltaban la importancia que tenía el manejo de las fuentes históricas, y en particular la Fundación Española de Historia Moderna destacaba el interés que debía tener en el perfil de los graduados en Historia la "capacidad de manejo de fuentes originales históricas, documentales o de otra índole (iconográficas, arqueológicas, etc.), expresadas en la lengua propia o en sus antecedentes históricos, y su conversión en materiales científicos mediante transcripción, resumen, catalogación, clasificación, comentario crítico y valoración” ${ }^{20}$. Los especialistas en Historia Contemporánea, en cambio, consideraban que el conocimiento de ciencias como la Paleografía y la Epigrafía, e incluso la Arqueología, eran competencias que pertenecían a “perfiles más específicos y especializados” y proponían que se incluyeran en el plan de estudios del futuro Grado en Historia como asignaturas obligatorias u optativas de cada universidad, “en vez de ponerlos como con-

18 J. A. CATALÁ et al., Informe para el diseño..., p. 91. Conviene recordar que en el Libro Blanco se solicitaba la valoración de las competencias en relación con los potenciales perfiles profesionales de la titulación: Enseñanza, Gestión del Patrimonio, Arqueología, Investigación, Archivos y Bibliotecas, etc. Sin embargo, como han criticado algunos colegas, la horquilla de estos potenciales perfiles profesionales era demasiado amplia y se incluyeron algunos que, con un mínimo de sentido común, deberían haberse excluido de las encuestas, C. GARCÍA MONERRIS, F. GÓMEZ OCHOA, “Los estudios universitarios de historia...”, pp. 309-310.

19 J. A. CATALÁ et al., Informe para el diseño..., pp. 127-141.

${ }^{20}$ J. A. CATALÁ et al., Informe para el diseño..., p. 134. 
tenidos comunes obligatorios (salvo que se trate de un reparto por áreas de conocimiento y no de las exigencias de un título "generalista”)"21.

En cualquier caso, por encima de los diferentes posicionamientos historiográficos o, incluso, de los intereses corporativos de las diferentes áreas de conocimiento, los redactores del Libro Blanco, establecieron cinco objetivos fundamentales que debía alcanzar el futuro Grado en Historia. Y entre ellos se destacaba que "el título de Historia debe proporcionar un conocimiento básico de los métodos, técnicas e instrumentos de análisis principales del historiador, lo que entraña, por un lado, la capacidad de examinar críticamente cualquier clase de fuentes y documentos históricos y, por otro, la habilidad de manejar los medios de búsqueda, identificación, selección y recogida de información, incluidos los recursos informáticos, y de emplearlos para el estudio y la investigación”22.

En lo que se refiere a la estructura general del futuro título, el Libro Blanco incluía el conocimiento y manejo de ciencias como la Epigrafía y Numismática entre los contenidos comunes obligatorios, enfatizando además que "resulta indispensable -aún tratándose del título de Grado- el contacto con la perspectiva de la investigación, esto es, con el ejercicio del oficio de historiador. A ello contribuye el contacto directo con las fuentes primarias y con los textos historiográficos producidos por la investigación, para lo que resulta necesario conocer, al menos de forma general, las técnicas de lectura de documentos antiguos en cualquier soporte suministradas por la Paleografía, la Epigrafía, la Numismática, etc.”23.

\section{DE LA TEORÍA A LA REALIDAD: LA EPIGRAFÍA Y NUMIS- MÁTICA EN EL GRADO EN HISTORIA.}

Pese a las reiteradas referencias que se hacen en el Libro Blanco sobre la importancia de la Epigrafía y Numismática, así como de otras ciencias historiográficas, en la formación de los futuros egresados, un análisis de los planes de estudio de los nuevos Grados en Historia permite comprobar que la realidad ha superado, si no la ficción, sí al menos la filosofía de aquel informe. De las 42 uni-

${ }^{21}$ J. A. CATALÁ et al., Informe para el diseño..., p. 140.

22 J. A. CATALÁ et al., Informe para el diseño..., p. 151. No hace falta enfatizar aquí que, detrás de la referencia a "cualquier clase de fuentes y documentos históricos" no se encuentran solo las fuentes utilizadas por los colegas de Historia Contemporánea, para las cuyo estudio no se requiere el dominio de ninguna ciencia historiográfica ni de ninguna lengua antigua, sino también aquellas fuentes escritas y materiales que son objeto de estudio por parte de los historiadores de la Antigüedad y medievalistas.

${ }^{23}$ J. A. CATALÁ et al., Informe para el diseño..., p. 157. 
versidades que en la actualidad ofertan el nuevo título, solo 24 incluyen la enseñanza de la Epigrafía y Numismática en sus planes de estudio. El resto, que supone casi el $43 \%$ del total, ha excluido la enseñanza de estas ciencias de la formación de los futuros historiadores (Tabla 1).

Así, solo en tres casos la asignatura se mantiene con la misma denominación que tenía en los antiguos planes de estudio, “Paleografía, Diplomática, Epigrafía y Numismática” (Universidad Autónoma de Madrid, Universidad a Distancia de Madrid de Madrid y Universidad Nacional a Distancia). En los tres casos se trata de una asignatura de 6 créditos ECTS de los que, en buena lógica, la mitad de ellos corresponden a la enseñanza de la Epigrafía y Numismática. Además, en la UDIMA y en la UNED esta asignatura es obligatoria, mientras que en la Universidad Autónoma de Madrid es una asignatura optativa. La Universidad de Alcalá de Henares, por su parte, ha cambiado la denominación de esta asignatura por "Introducción al estudio de los testimonios escritos", una asignatura de 6 créditos ECTS de carácter obligatorio, en la que se incluyen los contenidos de Paleografía y Epigrafía. En la Universidad de Alicante, a ambas materias le corresponde una cuarta parte de los 6 créditos ECTS de la asignatura obligatoria denominada "Metodología I”, lo que ha reducido notablemente la carga lectiva con respecto a la antigua Licenciatura en Historia.

Más acorde con las recomendaciones recogidas en el Libro Blanco es la situación de ambas ciencias en las 18 universidades que han incluido en sus planes de estudio del Grado en Historia una asignatura exclusiva para ambas materias, de 6 créditos ECTS (Tabla 1). En trece universidades la asignatura se ha denominado “Epigrafía y Numismática” y solo en cuatro se ha optado por otras variaciones en su denominación: "Fuentes escritas y numismáticas” (Universidad Complutense de Madrid), “Epigrafía y Numismática del Mundo Antiguo” (Universidad de las Islas Baleares), “Epigrafía y Numismática clásicas” (Universidad de Málaga) e “Inscripciones y monedas de la Antigüedad” (Universidad de Valladolid). Respecto a la naturaleza de estas asignaturas, en unos casos han sido consideradas como una asignatura de formación básica (Universidad de Almería, Universidad de Barcelona, Universidad de Cádiz y Universidad de Granada); en otros como una asignatura obligatoria (Universidad Complutense de Madrid, Universidad de Córdoba, Universidad de Extremadura, Universidad de Las Palmas de Gran Canaria, Universidad de Oviedo y Universidad del País Vasco); y en otras como optativa (Universidad Autónoma de Barcelona, Universidad de Cantabria, Universidad de Castilla-La Mancha, Universidad de Málaga y Universidad de Valladolid). 


\section{MANUEL RAMÍREZ SÁNCHEZ \\ LA DOCENCIA UNIVERSITARIA DE LA EPIGRAFÍA Y NUMISMÁTICA...}

Sin duda, la consideración de estas asignaturas como optativas reduce su potencial en la captación de estudiantes. Y en algunos casos también, según el itinerario donde éstas hayan sido ubicadas, el perfil de los mismos. Por ejemplo, en la Universidad Autónoma de Barcelona "Epigrafía y Numismática” se incluye como optativa solo en uno de los itinerarios que conduce a una de las nueve menciones del Grado en Historia, concretamente en la mención de Patrimonio Bibliográfico y Documental, y no se incluye como optativa en los itinerarios que conducen a las menciones de Historia Antigua o de Historia Medieval ${ }^{24}$. En otras universidades con menor número de itinerarios esta situación no se produce. Es el caso, por ejemplo, de la Universidad de Cantabria, en la que "Epigrafía y Numismática aparece como asignatura optativa en el itinerario de Historia aplicada, dentro de la materia de Técnicas de análisis documental, en la que también se incluye la asignatura optativa "Depósitos de la Cultura Escrita; Archivos y Bibliotecas", también de 6 créditos ECTS. Es similar la situación de la Universidad de Castilla-La Mancha, que oferta la asignatura como optativa dentro del itinerario de Patrimonio Histórico, en el que también se incluyen materias de Arqueología, Historia Antigua, Fuentes documentales y Patrimonio Histórico ${ }^{25}$.

En la actualidad, la universidad que incluye un mayor número de asignaturas de estas materias en el nuevo Grado en Historia es la Universidad Complutense de Madrid ${ }^{26}$. Además de la asignatura obligatoria "Fuentes escritas y numismáticas”, de 6 créditos ECTS, que se oferta en el tercer curso, incluye varias optativas para los distintos itinerarios del nuevo título. Así, los estudiantes que realizan el itinerario de Historia Antigua, por ejemplo, pueden escoger "Historia de la Escritura Latina” o "Numismática”, ambas del itinerario de Ciencias y Técnicas Historiográficas. Sin embargo, los alumnos que realizan el itinerario de Historia Me-

${ }^{24}$ Como los estudiantes se ven obligados a cursar cinco optativas vinculadas a un itinerario para que puedan obtener una mención, se imposibilita que un estudiante que desee obtener la mención en Historia Antigua por esta universidad pueda escoger la optativa de "Epigrafía y Numismática”. La misma situación se produce con las asignaturas optativas "Diplomática” o “Codicología y Paleografía”, vinculadas al itinerario de Patrimonio Bibliográfico y Documental, y los estudiantes que deseen obtener la mención de Historia Medieval.

${ }^{25}$ El Grado en Historia de la Universidad de Castilla-La Mancha, que se imparte en el Campus de Ciudad Real, tiene tres itinerarios curriculares, cada uno de los cuales conduce a la correspondiente mención en el título: Patrimonio Histórico, Historia del Mundo Hispánico y Antropología Cultural.

${ }^{26}$ El Grado en Historia posee siete itinerarios, que conducen a otras tantas menciones en el título: Historia Antigua; Historia Medieval; Historia Moderna; Historia Contemporánea; Antropología de América; Historia de América; y Ciencias y Técnicas Historiográficas. Los estudiantes que realizan algunos itinerarios pueden escoger entre una y tres optativas de otros itinerarios. 
dieval, pese a contar con una optativa de "Numismática" en el mismo, no pueden matricularse en "Historia de la Escritura Latina”, ya que este itinerario, al igual que sucede con el de Historia Moderna o el propio de Ciencias y Técnicas Historiográficas, no permite la posibilidad de que se cursen optativas de otros itinerarios.

Finalmente, dieciocho universidades que ofertan el Grado en Historia no incluyen en su plan de estudios ninguna asignatura de Epigrafía y Numismática, ni siquiera materias con otra denominación en las que se incluye la enseñanza de estas ciencias. Estas son, por orden alfabético, las siguientes: Universidad de Burgos, Universidad de Girona, Universidad de Jaén, Universidad Jaume I, Universidad de La Laguna, Universidad de La Rioja, Universidad de León, Universidad de Murcia, Universidad de Navarra, Universidad Pablo de Olavide, Universidad Rey Juan Carlos, Universidad de Santiago de Compostela, Universidad San PabloCEU, Universidad de Sevilla, Universidad de Valencia y Universidad de Zaragoza.

Algunas de estas universidades ofertan por vez primera este título y, en su mayoría, no cuentan con profesorado especializado en la docencia e investigación en Epigrafía y Numismática, lo que podría justificar la ausencia de estas materias en el plan de estudios del nuevo Grado ${ }^{27}$. Sin embargo, llama la atención que entre estas universidades se encuentran instituciones como las de Alicante, Sevilla, Valencia o Zaragoza, que cuentan con una dilatada tradición docente en estas materias y con un profesorado de reconocido prestigio en la docencia e investigación en Epigrafía y Numismática. En este último caso, la ausencia de estas materias en el plan de estudios se ha debido a que, en el largo proceso de discusión y negociación de los planes de estudio en las respectivas facultades, no han logrado ser aceptadas por encima de otras asignaturas que han contado con mayor apoyo por las distintas áreas de conocimiento. Y ello a pesar del empeño que han puesto en conseguirlo quienes, hasta la fecha, han venido encargándose de la docencia de estas asignaturas.

${ }^{27}$ Es el caso, por ejemplo, de las universidades de Burgos, Jaén, Jaume I o San Pablo-CEU. Esta última oferta desde el curso 2010-2011, por primera vez, el Grado en Historia. En su plan de estudios no se incluye la asignatura "Epigrafía y Numismática”, así como tampoco "Paleografía y Diplomática”. El Grado en Historia de la Universidad San Pablo-CEU aún no ha sido publicado en el Boletín Oficial del Estado, aunque ha pasado el trámite de su aprobación previa por el Consejo de Ministros. Cfr. Resolución de 22 de septiembre de 2009, de la Secretaría General de Universidades, por la que se publica el Acuerdo de Consejo de Ministros de 4 de septiembre de 2009, por el que se establece el carácter oficial de determinados títulos de Grado y su inscripción en el Registro de Universidades, Centros y Títulos (BOE n 244, de 9 de octubre de 2009). 


\section{LA ENSEÑANZA DE LA EPIGRAFÍA Y NUMISMÁTICA Y LAS LENGUAS CLÁSICAS.}

Como hemos explicado, las sucesivas reformas de los planes de estudio de las antaño denominadas Licenciaturas en Geografía e Historia, posteriormente de Historia (en aquellas Facultades que optaron por separar la enseñanza de ambas especialidades en dos títulos diferentes) y, finalmente, el diseño de los nuevos Grados en Historia, han traído consigo la desaparición de estas enseñanzas en algunas universidades, cuando no su inclusión en materias de contenido diverso, impartidas por profesorado de distintas especialidades, vinculados a diferentes áreas de conocimiento. Frente a la aparente uniformidad de los planes de estudio de la extinguida Licenciatura en Historia, que incluía la enseñanza de la Epigrafía y Numismática junto con la de la Paleografía y Diplomática, asistimos ahora a un nuevo panorama no solo debido a la supervivencia o desaparición es estas materias en los planes de estudio, sino incluso a su ubicación en uno u otro curso, en aquellas universidades en las que estas asignaturas han logrado permanecer.

Si en las anteriores licenciaturas en Historia estas asignaturas estaban situadas en el segundo ciclo de la titulación, por lo general en el tercer curso, en los nuevos grados podemos encontrar algunos ejemplos en los que la asignatura “Epigrafía y Numismática” se imparte en el segundo curso (Tabla 1). Si tradicionalmente ha sido un reto enseñar Epigrafía latina a muchos estudiantes que no han visto el Latín o el Griego en su paso por la Secundaria o incluso a quienes, habiéndolos estudiado, han olvidado casi por completo los rudimentos de estas lenguas, imaginemos cuán complicado puede ser la enseñanza de la Epigrafía y Numismática a los estudiantes de los nuevos Grados en Historia, que deben cursar esta asignatura cuando aún no han superado la Historia Antigua de Grecia y Roma, o la Historia Medieval ${ }^{28}$.

El Libro Blanco del Grado en Historia destacaba la necesidad de incluir el conocimiento de lenguas antiguas entre los contenidos instrumentales obligatorios u optativos del nuevo título, particularmente aquellas sobre las que se asienta la tradición cultural occidental: latín y griego ${ }^{29}$. Sobre este particular, un examen de la heterogénea muestra de los planes de estudio finalmente aprobados por cada

${ }^{28} \mathrm{O}$ cuando, en el menos malo de los escenarios, deben simultanear en el mismo curso, el aprendizaje de estas materias.

${ }^{29}$ En concreto, al ocuparse de la estructura del título se señalaba que “debería favorecerse en mayor grado el conocimiento del latín, ya que su uso abarca las edades antigua, media y moderna, sin que ello suponga negar la importancia del conocimiento del griego”. J. A. CATALÁ et al., Informe para el diseño..., p.162. 
universidad, y refrendados por la ANECA y el Ministerio, permite comprobar que la sugerencia cayó en saco roto en la mayoría de los casos, salvo honrosas excepciones.

Así, algunas universidades, como la de Granada, han incluido entre las materias de carácter básico del primer curso del Grado en Historia una asignatura denominada "Lengua Clásica”, de 6 créditos ECTS, lo que permite a los estudiantes ampliar sus conocimientos en Latín para que puedan cursar con mejores garantías las materias de formación básica correspondientes al bloque metodológico que se imparten en el segundo curso, particularmente la "Epigrafía y Numismática" ${ }^{\text {30 }}$. La misma situación se produce en el Grado en Historia de la Universidad de Oviedo, aunque en este caso la asignatura de primer curso, también de formación básica, se denomina "Introducción al Latín Clásico y Medieval”’31. La Universidad de Cantabria, en cambio, incluye una "Lengua Latina" como asignatura optativa de profundización en Historia, de 6 créditos ECTS, en el $4^{\circ}$ curso, permitiendo así su realización a los estudiantes que escojan la optativa "Epigrafía y Numismática”. La Universidad del País Vasco incluye la asignatura "Latín para historiadores” como obligatoria de 6 créditos ECTS, en el primer semestre del tercer curso, lo que permite a los estudiantes mejorar su preparación en esta lengua para superar la asignatura "Epigrafía y Numismática”, que se imparte en el segundo semestre del mismo curso. Por su parte, la Universidad de Salamanca oferta en el primer semestre del segundo curso una asignatura básica, de 6 créditos ECTS, denominada "Latín para historiadores", que sin duda será de gran utilidad para todos los estudiantes que cursen las asignaturas de Paleografía y Diplomática, Paleografía Medieval e Historia de la Escritura y Epigrafía y Numismática ${ }^{32}$.

30 En la materia "Lengua Clásica” los estudiantes pueden escoger entre las siguientes asignaturas: "Introducción a la Gramática Griega”, "Hebreo Bíblico”, “Árabe Clásico” (nivel 1) y “Lengua Latina” I. Cfr. Resolución de 16 de mayo de 2011, de la Universidad de Granada, por la que se publica el plan de estudios de Graduado en Historia (BOE $n^{0} 128$, de 30 de mayo de 2011).

${ }^{31}$ Como se señala en la Guía Docente de la asignatura, ésta destaca "su enfoque introductorio, con el que se pretende que los estudiantes se inicien- si no lo han hecho durante sus estudios de Bachillerato- y alcancen un nivel básico de conocimiento de la lengua latina, indispensable para desarrollar las competencias que requieren otras asignaturas de carácter obligatorio que forman parte del Grado en Historia, a saber: Epigrafía y Numismática, Arqueología clásica, Arqueología tardoantigua y medieval, Paleografía y Diplomática de la Edad Media y Paleografía y Diplomática de la Edad Moderna, así como las que integran la Materia "Técnicas de investigación y documentación históricas”, dentro del Bloque Optativo que se cursa durante $3^{\circ}$ y $4^{\circ}$ curso del Grado en Historia”.

${ }^{32}$ La asignatura de Latín, aunque se considera asignatura de formación básica, debe ser elegida por los estudiantes, junto con otra asignatura más, de entre las siguientes: "Introducción a la Sociología”; “Introducción a la Antropología”; “Lengua Moderna: Inglés académico”; y "Latín para historiadores”. Cfr. Resolución de 17 de marzo de 2011, de la Universidad de Salamanca, por 


\section{MANUEL RAMÍREZ SÁNCHEZ \\ LA DOCENCIA UNIVERSITARIA DE LA EPIGRAFÍA Y NUMISMÁTICA...}

También la Universidad de Vigo incluye una Lengua Clásica como materia de formación básica, en el primer semestre del primer curso, cuya utilidad será puesta en valor en la asignatura obligatoria de Paleografía, en el primer semestre del $2^{\circ}$ curso del Grado, así como en la asignatura "Epigrafía y Numismática”, optativa del primer semestre del $4^{\circ}$ curso del Grado. Finalmente, la Universidad de Valladolid oferta dos asignaturas introductorias al latín, como optativas de 6 créditos ECTS cada una de ellas, lo que garantiza la posibilidad de ampliar sus conocimientos en esta lengua a los futuros estudiantes de las dos asignaturas obligatorias de Paleografía y Diplomática, así como a los que elijan la asignatura optativa “Inscripciones y Monedas de la Antigüedad”, en el último curso del grado.

En cambio, algunas universidades se han visto obligadas a encajar la asignatura de Lenguas Clásicas que, hace años, estaba ubicada en el primer curso de la Licenciatura, en los últimos años del Grado, una vez los estudiantes han cursado la materia "Epigrafía y Numismática”. Es el caso, por citar un ejemplo, del grado en Historia de la Universidad de Córdoba que, con mucho acierto, ha incluido una materia obligatoria denominada "Lenguas Clásicas para el estudio de la Historia" pero que, sin embargo, se oferta en el segundo cuatrimestre del tercer curso, cuando la asignatura “Epigrafía y Numismática” se oferta en el primer cuatrimestre del segundo curso.

Algunas universidades de reciente creación, han sabido comenzar con mejor pie que otras con más larga tradición. Así, la Universidad a Distancia de Madrid, una universidad virtual de carácter privado, incluye en su grado en Historia una asignatura optativa denominada "Latín para historiadores", que se oferta en el segundo semestre del $2^{\circ}$ curso. Ello facilita a aquellos estudiantes que deseen ampliar sus conocimientos sobre esta lengua, o incluso aquellos que deseen iniciarse en la misma, la formación en Latín, permitiendo así un mejor aprovechamiento de la asignatura "Paleografía, Diplomática, Epigrafía y Numismática”, de 6 créditos ECTS, que se imparte en el segundo semestre del curso siguiente. Por su parte, la Universidad de La Rioja incluye en el plan de estudios del nuevo grado en Geografía e Historia una asignatura de formación básica, de 6 créditos ECTS, denominada "Lengua y literatura clásicas", que se imparte en el primer curso. Sin embargo, paradójicamente, no incluye en su plan de estudios la asignatura "Epigrafía y Numismática”33.

la que se publica el plan de estudios de Graduado en Geografía e Historia (BOE nº87, de 12 de abril de 2011).

${ }^{33} \mathrm{Ni}$ tampoco incluye las materias Paleografía y Diplomática. Cabe imaginar que estos contenidos deben estar incluidos en las asignaturas optativas del cuarto curso "Fuentes y métodos 
Sin embargo, la mayoría de las universidades, entre las cuales se incluye la universidad a la que está vinculado quien escribe, ni siquiera han considerado oportuno incluir una asignatura introductoria a las Lenguas Clásicas, ni siquiera como optativa, en el nuevo grado en Historia. Esperemos que en los futuros reajustes de estos títulos estas deficiencias puedan subsanarse, pero mientras tanto quienes impartimos estas materias deberemos afrontar con imaginación los mecanismos para solventar estos obstáculos.

\section{CONCLUSIONES.}

Hace ahora más de un siglo, el historiador Rafael Altamira publicaba un artículo en el Bulletin Hispanique en el que reflexionaba sobre el calado de la reforma ministerial que, por aquellos años, se estaba efectuando y que afectaba directamente a las facultades universitarias de Filosofía y Letras ${ }^{34}$. Se felicitaba Altamira por el carácter independiente que los estudios históricos habían alcanzado, pero también porque, por vez primera, las entonces consideradas ciencias instrumentales y auxiliares de la historia -"sin las que es imposible crear investigadores”, como señalaba el propio historiador-, así como los trabajos personales de seminario, alcanzaban el desarrollo que, unos años antes, había solicitado en su libro La enseñanza de la historia ${ }^{35}$. Una vez concluido el proceso de convergencia de nuestro sistema universitario con el Espacio Europeo de Educación Superior, los historiadores españoles tenemos la oportunidad de mirar atrás y comprobar qué aspectos positivos para nuestras disciplinas ha traído consigo la tan traída y llevada reforma, a la vez que podemos hacer inventario de aquellos efectos negativos que, a manera de "efectos colaterales", se han evidenciado en nuestros planes de estudios.

El primer balance es, sin lugar a dudas, positivo, toda vez que la enseñanza universitaria de la Historia se ha extendido en el sistema universitario español, al

de la Historia medieval” y “Fuentes, métodos y nuevas tendencias en Historia Moderna”. Cfr. Resolución de 15 de julio de 2010, de la Universidad de La Rioja, por la que se publica el plan de estudios de Graduado en Geografía e Historia (BOE $n^{\circ} 186$, de 2 de agosto de 2010).

${ }^{34}$ R. ALTAMIRA, "La reforma de los estudios históricos en España”, Bulletin Hispanique, 2, nº (1900), pp. 305-310.

${ }^{35}$ R. ALTAMIRA, La enseñanza de la Historia, Museo Pedagógico de Instrucción Primaria, Madrid, 1891. Existe una segunda versión, mucho más completa, editada por la Librería de Victoriano Suárez en 1895. Hace unos años, Ediciones Akal reeditó esta obra, con un excelente estudio preliminar de Rafael Asín Vergara, cfr. R. AltAMIRA, La enseñanza de la Historia, Akal, Madrid, 1997 ( $2^{\text {a }}$ edic.), pp. 13-78. Para las citas in extenso de las referencias de Rafael Altamira se ha procedido a normalizar la ortografía. 


\section{MANUEL RAMÍREZ SÁNCHEZ \\ LA DOCENCIA UNIVERSITARIA DE LA EPIGRAFÍA Y NUMISMÁTICA...}

pasar de las 34 universidades que impartían el título de Licenciado en Historia en 2004 a las 42 que, en el curso 2010-2011, ofertan el Grado en Historia a través de diversas variantes en la denominación del título: Historia, Geografía e Historia, Historia y Patrimonio, e Historia y Patrimonio Histórico. Igualmente positivo, o al menos esperanzador, parecía el horizonte que se planteaba a comienzos del debate de la reforma, en la elaboración del informe del Tuning Project (iniciado en 2003) y en las discusiones generadas en el proceso impulsado por la Comisión de Evaluación del diseño del Título de Grado en Historia, que condujeron a la redacción del llamado Libro Blanco ${ }^{36}$.

Sin embargo, cuando analizamos con detenimiento el resultado final del proceso, a través de la estructura de los diferentes planes de estudios del nuevo Título, así como en la definición de las asignaturas en determinados ámbitos o especialidades, podemos comprobar en qué medida las relaciones de poder y los lobbies académicos en algunas universidades han terminado por vulnerar la filosofía del Libro Blanco y, lo que es más grave aún, han hecho oídos sordos de algunas de las recomendaciones recogidas en dicho documento. En cualquier caso, más grave aún ha sido el hecho de que la ANECA, que fue quien encargó la elaboración del referido documento, haya procedido a verificar dichos títulos. Ahora más que nunca conviene recordar las palabras de Rafael Altamira a propósito de la reforma de los estudios históricos en España, a fines del siglo XIX:

Hay, en efecto, más de una opinión respetable que sostiene la necesidad de dar, v. gr. a las enseñanzas de Paleografía y Diplomática un carácter distinto según se destinen como auxiliares del trabajo personal a todos los estudiantes de Historia, o como ciencias sustantivas a los especialistas. No se puede, en rigor, pedir a todos los historiadores que sean paleógrafos, epigrafistas o numismáticos perfectos, porque cada uno de estos órdenes de conocimiento basta para absorber una vida entera, sin dejar tiempo para ninguna otra cosa; pero si en la Facultad no se estudian con todo el desarrollo de que son susceptibles, ¿dónde se formarán los especialistas, indispensables para el progreso de la ciencia general histórica? ${ }^{37}$

Transcurridos más de cien años desde que Rafael Altamira publicara estas reflexiones, parece que seguimos en el mismo punto del debate. Es evidente que, a la luz de la situación actual de la enseñanza de la Epigrafía y Numismática en los planes de estudio de los nuevos grados en Historia, en algunas universidades no solo se han obviado las recomendaciones del Libro Blanco de la ANECA sino que se han ignorado los esfuerzos de los historiadores que, antes y después de Altami-

\footnotetext{
${ }^{36}$ J. A. CATALÁ et al., Informe para el diseño...

${ }^{37}$ R. ALTAMIRA, La enseñanza de la Historia..., pp. 308-309.
} 
ra, tanto han hecho por la mejora de la enseñanza de la Historia en nuestras universidades y, en particular, por el desarrollo de la docencia e investigación de la Epigrafía y Numismática ${ }^{38}$. Esperamos que esta situación se corrija en las futuras revisiones de los planes de estudio y que estas líneas, al menos, hayan servido para abrir el debate entre los especialistas.

${ }^{38}$ Sobre la concepción teórica y metodológica de la ciencia epigráfica en España, cfr. J. Ma DE NAVASCUÉS, El concepto de la Epigrafía. Consideraciones sobre la necesidad de su ampliación, Real Academia de la Historia, Madrid, 1953; J. DE SANTIAGO FERNÁNDEZ, "La Epigrafía: evolución conceptual y metodológica”, Documenta \& Instrumenta 1 (2004), pp. 203220; M. RAMÍREZ SÁNCHEZ, "El concepto de Epigrafía. Consideraciones sobre la necesidad de su ampliación, cincuenta años después”, Signo, 15 (2005), pp. 47-76. 
MANUEL RAMÍREZ SÁNCHEZ

LA DOCENCIA UNIVERSITARIA DE LA EPIGRAFÍA Y NUMISMÁTICA...

\begin{tabular}{|c|c|c|c|c|c|c|c|}
\hline Universidad & Título & Nombre asignatura & Tipo & $\begin{array}{c}\text { Cur- } \\
\text { so }\end{array}$ & $\begin{array}{c}\text { Se- } \\
\text { mestre }\end{array}$ & $\begin{array}{l}\text { Crédi- } \\
\text { tos }\end{array}$ & BOE \\
\hline Alcalá & Historia & $\begin{array}{l}\text { Introducción al estudio de los } \\
\text { testimonios escritos }\end{array}$ & OB & 3 & 1 & 6 & $\begin{array}{c}23 / 03 / \\
2010\end{array}$ \\
\hline Alicante & Historia & Metodología I & OB & 4 & 1 & 6 & $\begin{array}{c}\text { Sin } \\
\text { datos }\end{array}$ \\
\hline Almería & Historia & Epigrafía y Numismática & FB & 2 & 1 & 6 & $\begin{array}{c}01 / 11 / \\
2011\end{array}$ \\
\hline $\begin{array}{l}\text { Autónoma de } \\
\text { Barcelona }\end{array}$ & Historia & Epigrafía y Numismática & OP & 4 & 1 & 6 & $\begin{array}{c}\text { Sin } \\
\text { datos }\end{array}$ \\
\hline $\begin{array}{l}\text { Autónoma de } \\
\text { Madrid }\end{array}$ & Historia & $\begin{array}{l}\text { Paleografía, Diplomática, Epi- } \\
\text { grafía y Numismática }\end{array}$ & OP & 4 & 1 & 6 & $\begin{array}{c}01 / 02 / \\
2010\end{array}$ \\
\hline Barcelona & Historia & Epigrafía y Numismática & FB & 2 & 1 & 6 & $\begin{array}{c}10 / 04 / \\
2012 \\
\end{array}$ \\
\hline Burgos & $\begin{array}{c}\text { Historia y } \\
\text { Patrimo- } \\
\text { nio }\end{array}$ & No existe & - & - & - & - & $\begin{array}{c}05 / 07 / \\
2011\end{array}$ \\
\hline Cádiz & Historia & Epigrafía y Numismática & FB & 2 & 2 & 6 & $\begin{array}{c}\text { Sin } \\
\text { datos }\end{array}$ \\
\hline Cantabria & Historia & Epigrafía y Numismática & OP & 4 & 1 & 6 & $\begin{array}{c}\text { Sin } \\
\text { datos }\end{array}$ \\
\hline $\begin{array}{l}\text { Castilla-La } \\
\text { Mancha }\end{array}$ & Historia & Epigrafía y Numismática & $\mathrm{OP}$ & 4 & 1 & 6 & $\begin{array}{c}12 / 11 / \\
2010 \\
\end{array}$ \\
\hline $\begin{array}{l}\text { Complutense } \\
\text { de Madrid }\end{array}$ & Historia & $\begin{array}{c}\text { Fuentes escritas y numismáticas } \\
\text { Historia de la Escritura Latina } \\
\text { Numismática (Itinerario } \mathrm{H}^{\mathrm{a}} \\
\text { Medieval) } \\
\text { Numismática (Itinerario CC. Y } \\
\text { TT. HH.) }\end{array}$ & $\begin{array}{l}\text { OB } \\
\text { OP } \\
\text { OP } \\
\text { OP }\end{array}$ & $\begin{array}{c}3 \\
4 \\
3-4 \\
3-4\end{array}$ & $\begin{array}{l}1 \\
1 \\
\text { s. d. } \\
\text { s. d. }\end{array}$ & $\begin{array}{l}6 \\
6 \\
6 \\
6\end{array}$ & $\begin{array}{c}21 / 06 / \\
2010\end{array}$ \\
\hline Córdoba & Historia & Epigrafía y Numismática & OB & 2 & 1 & 6 & $\begin{array}{c}11 / 06 / \\
2011\end{array}$ \\
\hline $\begin{array}{l}\text { Distancia de } \\
\text { Madrid }\end{array}$ & Historia & $\begin{array}{l}\text { Paleografía, Diplomática, Epi- } \\
\text { grafía y Numismática }\end{array}$ & OB & 3 & 2 & 6 & $\begin{array}{c}05 / 02 / \\
2010\end{array}$ \\
\hline Extremadura & $\begin{array}{l}\text { Historia y } \\
\text { Patrimo- } \\
\text { nio Histó- } \\
\text { rico }\end{array}$ & Epigrafía y Numismática & OB & 3 & 1 & 6 & $\begin{array}{c}08 / 03 / \\
2010\end{array}$ \\
\hline Girona & Historia & No existe & - & - & - & - & $\begin{array}{c}\text { Sin } \\
\text { datos }\end{array}$ \\
\hline Granada & Historia & Epigrafía y Numismática & FB & 2 & 1 & 6 & $\begin{array}{c}16 / 05 / \\
2011\end{array}$ \\
\hline Huelva & Historia & No existe & - & - & - & - & $\begin{array}{c}17 / 05 / \\
2011\end{array}$ \\
\hline Illes Balears & Historia & $\begin{array}{c}\text { Epigrafía y Numismática del } \\
\text { Mundo Antiguo }\end{array}$ & OB & 4 & 1 & 6 & $\begin{array}{c}29 / 03 / \\
2010 \\
\end{array}$ \\
\hline Jaén & $\begin{array}{l}\text { Geografía } \\
\text { e Historia }\end{array}$ & No existe & - & - & - & - & $\begin{array}{c}22 / 02 / \\
2011\end{array}$ \\
\hline Jaume I & $\begin{array}{l}\text { Historia y } \\
\text { Patrimo- } \\
\text { nio }\end{array}$ & No existe & - & - & - & - & $\begin{array}{c}25 / 08 / \\
2011\end{array}$ \\
\hline La Laguna & Historia & No existe & - & - & - & - & $\begin{array}{c}24 / 06 / \\
2010\end{array}$ \\
\hline $\begin{array}{l}\text { Las Palmas } \\
\text { de Gran } \\
\text { Canaria }\end{array}$ & Historia & Epigrafía y Numismática & OB & 2 & 1 & 6 & $\begin{array}{c}24 / 12 / \\
2009\end{array}$ \\
\hline La Rioja & $\begin{array}{l}\text { Geografía } \\
\text { e Historia }\end{array}$ & No existe & - & - & - & - & $\begin{array}{c}15 / 06 / \\
2010\end{array}$ \\
\hline
\end{tabular}


MANUEL RAMÍREZ SÁNCHEZ

LA DOCENCIA UNIVERSITARIA DE LA EPIGRAFÍA Y NUMISMÁTICA...

\begin{tabular}{|c|c|c|c|c|c|c|c|}
\hline León & Historia & No existe & - & - & - & - & $\begin{array}{c}16 / 07 / \\
2010\end{array}$ \\
\hline Lleida & Historia & $\begin{array}{c}\text { Paleografía, Diplomática y } \\
\text { Epigrafía }\end{array}$ & OP & 4 & s. d. & 6 & $\begin{array}{l}07 / 03 / \\
2009\end{array}$ \\
\hline Málaga & Historia & $\begin{array}{c}\text { Epigrafía y Numismática clási- } \\
\text { cas }\end{array}$ & OP & 4 & 1 & 6 & $\begin{array}{l}18 / 04 / \\
2012 \\
\end{array}$ \\
\hline Murcia & Historia & No existe & - & - & - & - & $\begin{array}{c}15 / 07 / \\
2010\end{array}$ \\
\hline $\begin{array}{l}\text { Nacional a } \\
\text { Distancia }\end{array}$ & $\begin{array}{l}\text { Geografía } \\
\text { e Historia }\end{array}$ & $\begin{array}{c}\text { Paleografía, Diplomática, Epi- } \\
\text { grafía y Numismática }\end{array}$ & OB & 5 & 1 & 6 & $\begin{array}{c}27 / 07 / \\
2010\end{array}$ \\
\hline Navarra & Historia & No existe & - & - & - & - & $\begin{array}{c}08 / 02 / \\
2010\end{array}$ \\
\hline Oviedo & Historia & Epigrafía y Numismática & OB & 2 & 2 & 6 & $\begin{array}{c}15 / 07 / \\
2010\end{array}$ \\
\hline $\begin{array}{l}\text { Pablo de } \\
\text { Olavide }\end{array}$ & $\begin{array}{l}\text { Geografía } \\
\text { e Historia }\end{array}$ & No existe & - & - & - & - & $\begin{array}{c}\text { Sin } \\
\text { datos }\end{array}$ \\
\hline País Vasco & Historia & Epigrafía y Numismática & OB & 3 & 2 & 6 & $\begin{array}{c}31 / 01 / \\
2011\end{array}$ \\
\hline $\begin{array}{l}\text { Rey Juan } \\
\text { Carlos }\end{array}$ & Historia & No existe & - & - & - & - & $\begin{array}{c}01 / 12 / \\
2009\end{array}$ \\
\hline $\begin{array}{l}\text { Rovira i } \\
\text { Virgili }\end{array}$ & Historia & No existe & - & - & - & - & $\begin{array}{c}18 / 03 / \\
2011\end{array}$ \\
\hline Salamanca & Historia & Epigrafía y Numismática & OP & 3 & 2 & 6 & $\begin{array}{l}12 / 04 / \\
2011 \\
\end{array}$ \\
\hline $\begin{array}{l}\text { Santiago de } \\
\text { Compostela }\end{array}$ & Historia & No existe & - & - & - & - & $\begin{array}{c}05 / 03 / \\
2010\end{array}$ \\
\hline $\begin{array}{l}\text { San Pablo- } \\
\text { CEU }\end{array}$ & Historia & No existe & - & - & - & - & $\begin{array}{c}\text { Sin } \\
\text { datos }\end{array}$ \\
\hline Sevilla & Historia & No existe & - & - & - & - & $\begin{array}{c}20 / 01 / \\
2011\end{array}$ \\
\hline Valencia & Historia & No existe & - & - & - & - & $\begin{array}{c}30 / 12 / \\
2011\end{array}$ \\
\hline Valladolid & Historia & $\begin{array}{l}\text { Inscripciones y Monedas de la } \\
\text { Antigüedad }\end{array}$ & OP & 4 & s.d. & 6 & $\begin{array}{c}03 / 01 / \\
2011\end{array}$ \\
\hline Vigo & $\begin{array}{l}\text { Geografía } \\
\text { e Historia }\end{array}$ & Epigrafía y Numismática & OP & 4 & 1 & 6 & $\begin{array}{l}01 / 11 / \\
2010 \\
\end{array}$ \\
\hline Zaragoza & Historia & No existe & - & - & - & - & $\begin{array}{c}29 / 11 / \\
2010\end{array}$ \\
\hline
\end{tabular}

Tabla 1. La Epigrafía y Numismática en los nuevos Grados en Historia en las universidades españolas. Tipología de las asignaturas: $\mathrm{FB}=$ asignatura de formación básica; $\mathrm{OB}$ = asignatura obligatoria; $\mathrm{OP}$ = asignatura optativa (Elaboración propia). 